\title{
ESTUDO DAS CARACTERÍSTICAS FÍSICO-QUÍMICAS, MICROBIOLÓGICAS E SENSORIAIS DE EMBUTIDOS FERMENTADOS TIPO SALAME FORMULADOS COM DIFERENTES PROPORÇÕES DE CARNE CAPRINA E SUÍNA
}

\author{
RENATA TIEKO NASSU * \\ LIRENY APARECIDA GUARALDO GONÇALVES ** \\ FREDERICO JOSÉ BESERRA *** \\ TEREZINHA FEITOSA *
}

\begin{abstract}
Embutidos fermentados tipo salame foram formulados, com diferentes proporções de carne suína e carne caprina, visando o aproveitamento da carne de caprinos na forma de produto processado. A influência das diferentes formulações na evolução do $\mathrm{pH}$ e atividade de água (Aw) foi avaliada. Os produtos finais obtidos foram caracterizados quanto ao teor de gordura, proteína e umidade. Paralelamente, foram realizadas análises microbiológicas e de aceitação sensorial (mediante escala hedônica de 9 pontos). A incorporação de carne de caprinos nas formulações influenciou os valores finais de $\mathrm{pH}$ e Aw. Comparados com amostra comercial, os embutidos fermentados de carne suína/carne caprina apresentaram valores similares de gordura, enquanto que a umidade foi mais elevada para os produtos formulados. Os valores obtidos na contagem de microrganismos enquadraram-se nos padrões estabelecidos pela legislação em vigor. A formulação contendo $25 \%$ carne de caprinos e $75 \%$ carne de suínos foi considerada como ideal, sem apresentar interferência na aceitação sensorial do produto.
\end{abstract}

PALAVRAS-CHAVE: SALAME; CAPRINOS; SUÍNOS; COMPOSIÇÃO.

* Pesquisadoras, Embrapa Agroindústria Tropical, Fortaleza, CE.

** Profa. Dra., Laboratório de Óleos e Gorduras, Faculdade de Engenharia de Alimentos (FEA), Universidade Estadual de Campinas (UNICAMP), Campinas, SP.

*** Prof. Dr., Laboratório de Carnes, Departamento de Tecnologia de Alimentos (DTA), Centro de Ciências Agrárias (CCA), Universidade Federal do Ceará (UFC), Fortaleza, CE. 


\section{INTRODUÇÃO}

A caprinocultura consiste em atividade de expressão econômica no Nordeste brasileiro, o qual concentra cerca de $90 \%$ do rebanho nacional, sendo a Bahia, Piauí, Pernambuco e Ceará os maiores produtores.

A carne e o leite, provenientes de caprinos, representam renda para o pequeno produtor, podendo ser consumidos tanto na forma in natura como de produto processado: queijo, iogurte, carne salgada e seca, entre outros. O aproveitamento racional deste tipo de carne é necessário, pois direciona os cortes nobres para consumo e o restante para o processamento. Além disso, pode-se utilizar nestes processos a carne de animais velhos e/ou provenientes de descarte, visando melhor aproveitamento deste criatório. A carne proveniente de animais velhos ou de descarte pode ser incorporada em até $30 \%$ da formulação, substituindo a carne bovina (23). Os animais de descarte também podem ser aproveitados em embutidos cozidos, defumados e/ou fermentados como, por exemplo, salames (carnes bovina, suína e ovina/caprina, contendo toucinho), "krakauer" (embutido de carne ovina/caprina e suína), "lyoner" (produto de composição similar aos salames, porém sem sofrer fermentação) e salsichas tipo Viena (23).

O embutido cru fermentado (salame) é uma alternativa, pois além de ser estável em temperatura ambiente, o sabor ácido proporcionado pela presença de bactérias lácticas auxilia no mascaramento do sabor e aroma típicos destes animais.

SILVEIRA \& ANDRADE (21) recomendam a utilização de carne proveniente de animais mais velhos na formulação de produtos fermentados por apresentarem teor de umidade mais baixo e coloração mais acentuada.

Diversos autores relataram variações no tipo de matéria-prima para elaboração de embutidos fermentados. ACTON \& DICK (1) utilizaram carne de peru para produção de embutido fermentado e estudaram a influência de parâmetros como temperatura e porcentagem de gordura nas características sensoriais do produto. KLETTNER et al. (14) pesquisaram o processamento de salame a partir de misturas em partes iguais de carnes ovina, bovina e suína. Alguns estudos utilizando a carne de frango mecanicamente separada (CFMS) tiveram bons resultados em relação à qualidade organoléptica com adições de até 37\% de CFMS, não havendo influência na queda do $\mathrm{pH}$ durante o processamento com adições de até $25 \%(10,20)$. Em alguns países como a Noruega são produzidos embutidos fermentados secos com carne ovina (11). HWANG et al. (12) prepararam 
embutidos fermentados com carne de bacalhau, junto de carnes de frango, bovina ou suína. Os embutidos formulados com carne de bacalhau foram considerados como aceitáveis, porém alguns provadores perceberam o sabor de peixe nos embutidos mistos de carnes de peixe e de frango. BÖHME et al. (7) relataram a utilização de carne de avestruz na formulação de salame tipo Italiano utilizando diferentes culturas starters. Afirmaram que a melhor combinação para este tipo de carne seria composta por $L$. curvatus e Micrococcus sp., que resulta em salame com qualidades organolépticas aceitáveis.

ARGANOSA et al. (4) desenvolveram embutidos frescos formulados com diferentes proporções de carnes caprina e suína. Os valores de umidade, gordura e proteína variaram de acordo com a porcentagem de gordura da carne suína. Não foram encontradas diferenças significativas na textura, aceitação global e sabor estranho para os embutidos com diferentes níveis de carne de caprinos. KRUPA et al. (15) relataram o processamento de salsichas, cujas formulações continham carne de suínos e de caprinos. Os teores de umidade e proteína aumentaram enquanto que o teor de gordura diminuiu à medida que a porcentagem de carne caprina foi adicionada. Além disso, evidenciaram que a carne de caprinos constituise em matéria-prima econômica e de boa aceitação. Em outro estudo, DZUDIE \& TANDEM (9) compararam salsichas feitas a partir de carne de caprinos, coelhos e bovinos, quanto à sua composição, analisando as amostras sensorialmente. As salsichas de carne de caprinos obtiveram maiores pontuações em relação às de carne de bovinos. BERRY et al. (6) compararam produtos reestruturados elaborados com carne de caprinos e formulados com outros tipos de carnes e afirmaram que a rejeição aos produtos contendo carne de caprinos, aparentemente devido ao aroma e sabor característicos, poderiam ser revertidos com uso de carne bovina ou suína, em formulações mistas.

Este trabalho teve por objetivo avaliar as características físico-químicas, microbiológicas e sensoriais de embutido fermentado tipo salame, formulado com diferentes proporções de carne de suínos/caprinos.

\section{MATERIAL E MÉTODOS}

\subsection{PROCESSAMENTO DE EMBUTIDO FERMENTADO}

Carne de caprino, proveniente de animais adultos, sem distinção de raça, desossada da carcaça inteira, fornecida pela Embrapa Caprinos (Sobral - 
CE) e carne de suínos, desossada e congelada, adquirida no mercado local foram previamente moídas em discos de $8 \mathrm{~mm}$ e congeladas em freezer a $-18^{\circ} \mathrm{C}$, na forma de camadas finas de, aproximadamente, $2 \mathrm{~cm}$. Após o congelamento, as carnes foram quebradas em pequenos blocos e moídas em discos de $6 \mathrm{~mm}$, assim como a gordura (toucinho suíno). Foram utilizadas as seguintes proporções de carne suína/carne caprina: 0/100, 25/75, 50/50, 75/25 e 100/0\%. Os ingredientes utilizados foram: sal $(2,5 \%)$, mistura comercial para cura $(0,30 \%)$, isoascorbato de sódio $(0,25 \%)$, glicose $(0,50 \%)$, sacarose $(0,50 \%)$, glutamato monossódico $(0,20 \%)$, pimenta-branca $(0,10 \%)$, noz-moscada $(0,10 \%)$ e alho em pó $(0,10 \%)$. Foi utilizada cultura starter contendo os microrganismos Staphylococcus xylosus e Pediococcus pentosaceus, na proporção $0,02 \%$. A carne, a gordura e os ingredientes foram colocados em misturadeira e a seguir embutidas em tripas de colágeno reconstituído (calibre $45 \mathrm{~mm}$ ). As amostras foram levadas para câmara com umidade relativa e temperatura controladas, durante 14 dias (Tabela 1). Foram processadas duas bateladas de $3 \mathrm{~kg}$ de produto por tratamento.

\section{TABELA 1 - CONDIÇÕES DE PROCESSAMENTO (TEMPERATURA E UMIDADE RELATIVA) DE EMBUTIDO FERMENTADO DE CARNE DE CAPRINOS}

\begin{tabular}{c|c|c|c|c}
\hline \multirow{2}{*}{ DIA DE PROCESSO } & \multicolumn{2}{|c|}{ TEMPERATURA ( C) } & \multicolumn{2}{c}{ UMIDADE RELATIVA (\%) } \\
\cline { 2 - 5 } & M nima & MA区ima & M nima & MA区ima \\
\hline 1 & 22 & 23 & 85 & 95 \\
\hline 2 & 18 & 20 & 80 & 90 \\
\hline 3 em diante & 12 & 15 & 70 & 80 \\
\hline
\end{tabular}

Foram monitorados os valores de $\mathrm{pH}$ e atividade de água das amostras aos $0,1,2,7$ e 14 dias. As amostras obtidas ao final do processamento (14 dias) foram imediatamente analisadas.

\subsection{ANÁLISES FÍSICO-QUÍMICAS}

Para as análises físico-químicas foram coletados aleatoriamente três gomos de amostra para cada tratamento, sendo as análises realizadas em triplicata. Os embutidos fermentados foram submetidos às seguintes análises físico-químicas: umidade, proteína e gordura, segundo a AOAC (3); $\mathrm{pH}$, conforme técnica descrita por TERRA \& BRAUN (22) e atividade de água $(A w)$, utilizando-se aparelho medidor de atividade de água, marca Decagon Devices Inc., modelo Aqualab CX-2. 


\subsection{ANÁLISES MICROBIOLÓGICAS}

As análises microbiológicas, realizadas logo após o processamento, envolveram: coliformes a $45^{\circ} \mathrm{C}$, pesquisa de Salmonella e estafilococos coagulase positiva (2). Foram efetuadas as contagens de estafilococos coagulase positiva depois de 48 horas para verificação de desenvolvimento deste microrganismo, após o período de fermentação, também segundo metodologia da APHA (2).

\subsection{ANÁLISE SENSORIAL}

$\mathrm{Na}$ avaliação sensorial, efetuada por 30 provadores não-treinados, foi empregado teste de aceitação global utilizando-se escala hedônica estruturada de 9 pontos (desgostei muitíssimo = nota 1 e gostei muitíssimo = nota 9). Amostras com, aproximadamente, $10 \mathrm{~g}$, equivalentes a duas fatias de salame foram entregues aos provadores em pratos plásticos brancos, codificados com números de três dígitos, acompanhados de um copo de água e de biscoito tipo água e sal (para ser utilizado pelo provador entre as amostras). O número máximo de amostras por sessão para cada provador foi de três, entregues separadamente (apresentação monádica) (16).

Os resultados foram avaliados pela análise de variância e pelo teste de Tukey, ao nível de $5 \%$ de significância, mediante o programa estatístico SAS for Windows, versão 6.12 (19).

\section{RESULTADOS E DISCUSSÃO}

A evolução do pH e Aw durante o processamento das formulações mistas de embutido fermentado são apresentadas nas Figuras 1 e 2 . Todos os tratamentos apresentaram o mesmo comportamento, isto é, queda nos valores de $\mathrm{pH}$ e Aw no decorrer do tempo.

A incorporação da carne de caprinos, juntamente com a de suínos na formulação de embutido fermentado influenciou os valores finais de Aw e $\mathrm{pH}$ (Tabela 2). A diferença entre as matérias-primas em propriedades como força tamponante (capacidade de determinada substância em resistir à variação de pH) e capacidade de retenção de água deve ter ocasionado a alteração nos produtos finais. Quanto maior a força tamponante, maior a quantidade de ácido a ser produzida pelas bactérias para abaixar o pH do 
FIGURA 1 - EVOLUÇÃO DO pH DE EMBUTIDOS FERMENTADOS FORMULADOS COM DIFERENTES PROPORÇÕES DE CARNE DE SUÍNOS E DE CAPRINOS

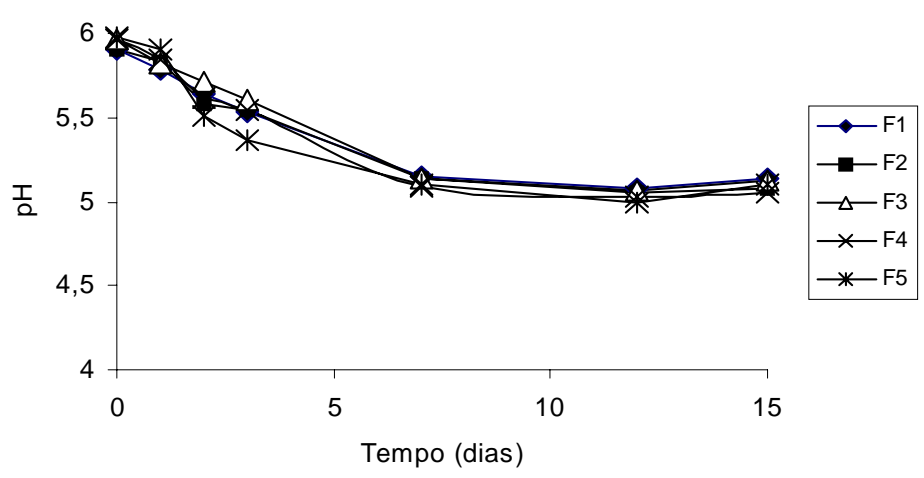

F1 - 100\% suíno; F2 - 25\% caprino/75\% suíno; F3 - 50 \% caprino/50\% suíno; F4 - 75\% caprino/25\% suíno; F5 - 100\% caprino.

FIGURA 2 - EVOLUÇÃO DA AW DE EMBUTIDOS FERMENTADOS FORMULADOS COM DIFERENTES PROPORÇÕES DE CARNE DE SUÍNOS E DE CAPRINOS

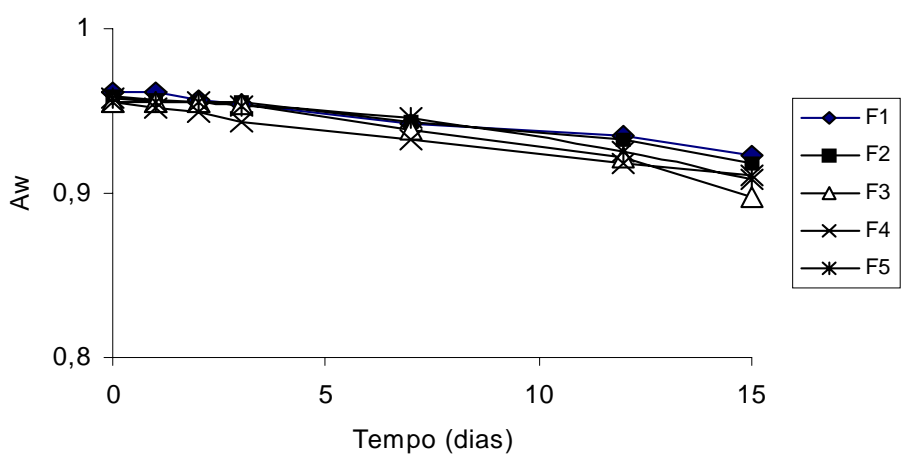

F1 = 100\% suíno; F2 = 25\% caprino/75\% suíno; F3 = 50\% caprino/50\% suíno; F4=- $75 \%$ caprino/25\% suíno; F5 = 100\% caprino. 
produto, resultando em fermentação mais lenta (5). Se a força tamponante da carne for muito baixa, como acontece em carnes tipo Pale, Soft, Exsudative (PSE), os produtos resultantes da fermentação dos açúcares produzem condições ácidas muito rapidamente, ocorrendo excesso de acidificação do produto final. Tal fato influi na estabilidade da cor do produto e prejudica o desenvolvimento de sabor e de aroma. Por outro lado, quando a força tamponante é muito alta, como no caso de carnes Dry, Firm, Dark (DFD) ocorrem problemas na secagem do produto, pois as proteínas retêm mais água, dificultando o processo. Carnes deste tipo também apresentam maior susceptibilidade a alterações microbiológicas $(5,8$, $13,18)$. Outro fator importante é o $\mathrm{pH}$ inicial das carnes que influencia o tempo de fermentação e o $\mathrm{pH}$ do produto final. Carnes com maiores valores de $\mathrm{pH}$ necessitam maior produção de ácido para alcançar o mesmo ponto final (5). Segundo BACUS (5) muitos processadores notaram que produtos com formulações mistas de carnes suína e bovina fermentam mais rapidamente que os que contêm apenas carne bovina. Várias hipóteses incluem maiores níveis de contaminação lática encontrados na carne suína e/ou maiores concentrações de tiamina. Além disso, carne bovina geralmente apresenta $\mathrm{pH}$ inicial mais alto e maior capacidade tamponante. A flora microbiana também deve ser considerada, principalmente devido às condições de manuseio, o que pode influenciar a fermentação. Neste estudo não foi considerado este efeito, uma vez que culturas iniciadoras foram utilizadas exatamente para minimizá-lo, entre outros motivos. As amostras apresentaram cerca de $30 \%$ de perda de peso.

\section{TABELA 2 - VALORES FINAIS DE pH E AW DE EMBUTIDOS FERMENTADOS FORMULADOS COM DIFERENTES PROPORÇÕES DE CARNE DE SUÍNOS E CAPRINOS}

\begin{tabular}{l|c|c|c|c|c}
\hline & \multicolumn{5}{|c}{ TRATAMENTO } \\
\cline { 2 - 6 } & $\mathbf{F 1}$ & F2 & F3 & F4 & F5 \\
\hline $\mathrm{pH}$ & $5,14 \mathrm{a}$ & $5,13 \mathrm{a}$ & $5,07 \mathrm{~b}$ & $5,05 \mathrm{~b}$ & $5,10 \mathrm{~b}$ \\
$\mathrm{Aw}$ & $0,923 \mathrm{a}$ & $0,918 \mathrm{a}$ & $0,897 \mathrm{~b}$ & $0,911 \mathrm{~b}$ & $0,908 \mathrm{~b}$ \\
\hline
\end{tabular}

Amostras seguidas pelas mesmas letras na mesma linha não diferem $(p>0,05)$ pelo teste de Tukey. F1 = 100\% suíno; F2 = 25\% caprino/75\% suíno; F3 = 50\% caprino/50\% suíno; F4 = 75\% caprino/25\% suíno; F5 = 100\% caprino.

Comparando-se as amostras de embutido fermentado de marca comercial e as amostras com carnes de suínos/caprinos (Tabela 3) observou-se que os valores de umidade foram mais elevados para as amostras processadas do que para a comercial. Este fato provavelmente se deve mais às condições de processamento dos produtos, tais como temperatura de maturação e fermentação, processo de secagem, do que à substituição 
da carne bovina pela de caprinos. Em relação aos valores de gordura, a amostra comercial apresentou teor de gordura similar aos produtos processados neste estudo.

\section{TABELA 3 - VALORES MÉDIOS DE COMPOSIÇÃO QUÍMICA DE EMBUTIDOS FERMENTADOS FORMULADOS COM DIFERENTES PROPORÇÕES DE CARNE DE SUÍNOS E CAPRINOS}

\begin{tabular}{|c|c|c|c|c|c|c|}
\hline & \multicolumn{5}{|c|}{ TRATAMENTO } & \multirow[b]{2}{*}{$\begin{array}{c}\text { Amostra } \\
\text { comercial }\end{array}$} \\
\hline & F1 & F2 & F3 & F4 & F5 & \\
\hline $\begin{array}{c}\text { Umidade } \\
(\%)\end{array}$ & $49,40 \pm 0,22$ & $49,24 \pm 0,37$ & $51,99 \pm 0,83$ & $47,14 \pm 0,14$ & $52,47 \pm 1,50$ & $36,13 \pm 0,48$ \\
\hline $\begin{array}{l}\text { Prote na } \\
(\%)\end{array}$ & $22,04 \pm 0,18$ & $22,47 \pm 0,45$ & $20,54 \pm 0,40$ & $22,96 \pm 0,25$ & $19,80 \pm 1,47$ & $26,75 \pm 0,70$ \\
\hline $\begin{array}{c}\text { Gordura } \\
(\%)\end{array}$ & $24,60 \pm 0,28$ & $22,26 \pm 0,73$ & $23,29 \pm 0,76$ & $25,28 \pm 1,38$ & $21,66 \pm 0,90$ & $25,32 \pm 0,22$ \\
\hline
\end{tabular}

1 Média de amostras de duas marcas diferentes de salame tipo italiano.

F1 = 100\% suíno; F2 = 25\% caprino/75\% suíno; F3 = 50\% caprino/ $50 \%$ suíno; F4 = 75\% caprino/25\% suíno; F5 = 100\% caprino.

As características microbiológicas dos produtos obtidos atenderam à legislação federal em vigor (Tabela 4).

\section{TABELA 4- CARACTERÍSTICAS MICROBIOLÓGICAS DE EMBUTIDOS FERMENTADOS FORMULADOS COM DIFERENTES PROPORÇÕES DE CARNE DE SUÍNOS E CAPRINOS}

\begin{tabular}{cccccc}
\hline & \multicolumn{5}{c}{ TRATAMENTOS } \\
\cline { 2 - 5 } & F1 & F2 & F3 & F4 & F5 \\
\cline { 2 - 5 } Coliformes a 45 C (NMP/g) & $<3$ & $<3$ & $<3$ & $<3$ & $<3$ \\
$\begin{array}{c}\text { Estafilococos coag. pos. } \\
\text { (UFC/g) }\end{array}$ & $<10$ & $<10$ & $<10$ & $<10$ & $<10$ \\
$\begin{array}{c}\text { Estafilococos coag. pos. } \\
\text { (48h) (UFC/g) } \\
\text { Salmonella sp/25 g }\end{array}$ & AusCficia & AusCffia & AusCficia & AusCffia & AusCffia
\end{tabular}

F1 = 100\% suíno; F2 = 25\% caprino/75\% suíno; F3 = 50\% caprino/50\% suíno; F4 = 75\% caprino/25\% suíno; F5 = 100\% caprino.

Em relação à análise sensorial, os valores médios de aceitação global variaram de 3,7 a 5,4, para as amostras formuladas com $100 \%$ de carne 
caprina e $25 \%$ de carne caprina $75 \%$ carne suína, respectivamente (Tabela 5). O baixo valor obtido para o embutido formulado apenas com carne caprina pode ser explicado, pois os provadores acabam avaliando as amostras de forma comparativa, mesmo quando apresentadas em ordem monádica. Assim, tendo como referência amostras com porcentagens diferentes de carne suína, o embutido fermentado formulado apenas com carne caprina apresentou menor aceitação.

\section{TABELA 5 - VALORES MÉDIOS DE ACEITAÇÃO GLOBAL DE EMBUTIDOS FERMENTADOS FORMULADOS COM DIFERENTES PROPORÇÕES DE CARNE DE SUÍNOS E CAPRINOS}

\begin{tabular}{cccccc}
\hline & \multicolumn{5}{c}{ TRATAMENTOS } \\
\cline { 2 - 6 } & F1 & F2 & F3 & F4 & F5 \\
\hline Aceita $\stackrel{\text { a o sensorial }}{5} 5,1 \mathrm{a}$ & $5,4 \mathrm{a}$ & $4,7 \mathrm{~b}$ & $4,2 \mathrm{bc}$ & $3,7 \mathrm{c}$ \\
\hline
\end{tabular}

Amostras seguidas pelas mesmas letras na mesma linha não diferem $(p>0,05)$ pelo teste de Tukey.

F1 = 100\% suíno; F2 = 25\% caprino/75\% suíno; F3 = 50\% caprino/50\% suíno; F4 = 75\% caprino/25\% suíno; F5 = 100\% caprino.

Os valores de aceitação global entre o tratamento com $100 \%$ de carne suína e o com $25 \%$ carne caprina/ $75 \%$ carne suína não apresentaram diferença significativa ( $p>0,05)$. O somatório das notas $6 / 7 / 8 / 9$ da escala hedônica demonstra que a amostra com $25 \%$ carne caprina/ $75 \%$ carne suína apresentou a maior porcentagem de notas entre 6 e 9 (Figura 3). Analisando a freqüência das notas para cada amostra (Figura 4) pode-se observar que amostras com maior porcentagem de carne caprina receberam maior freqüência de notas abaixo de 5 , enquanto que aquelas com menor porcentagem de carne caprina apresentaram maior freqüência de notas acima de 6 . Tal fato pode ser comprovado pelo comportamento da amostra com $25 \%$ carne caprina/ $75 \%$ carne suína.

KLETTNER et al. (14) relataram a adição de até 33\% de carne de ovinos velhos, junto com carnes suína e bovina na formulação de produtos cárneos entre os quais embutido fermentado, sem que os provadores detectassem a presença de carne ovina na avaliação sensorial. MELO (17) também estudou a utilização de carne de caprinos na elaboração de embutido cozido, tipo apresuntado. A formulação com $25 \%$ de carne de caprino e $75 \%$ de carne de suíno foi a melhor avaliada, de acordo com o teste de aceitação sensorial conduzido. 
FIGURA 3 - REPRESENTAÇÃO GRÁFICA DO SOMATÓRIO DAS FREQÜÊNCIAS DE NOTAS 6/7/8/9 DA ESCALA HEDÔNICA DE EMBUTIDOS FERMENTADOS FORMULADOS COM DIFERENTES PROPORÇÕES DE CARNE DE SUÍNOS E CAPRINOS

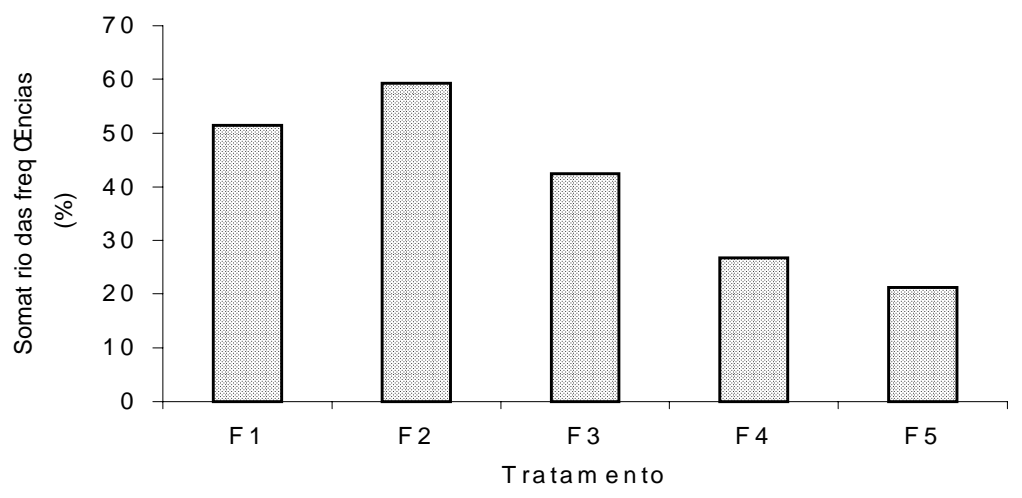

F1 = 100\% suíno; F2 = 25\% caprino/75\% suíno; F3 = 50\% caprino/50\% suíno; F4 = 75\% caprino/25\% suíno; F5 = 100\% caprino.

FIGURA 4 - HISTOGRAMA DE FREQÜÊNCIA PARA NOTAS DE ACEITAÇÃO GLOBAL DOS PRODUTOS FORMULADOS COM DIFERENTES PROPORÇÕES DE CARNE DE SUÍNOS ECAPRINOS

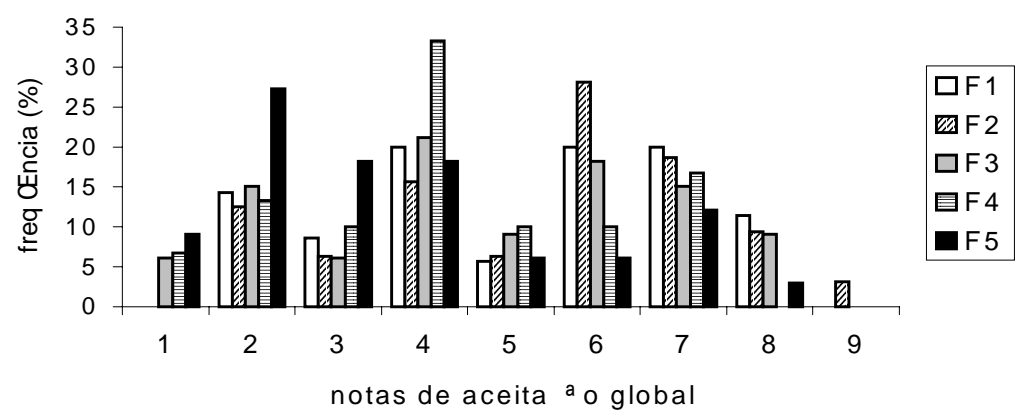

F1 = 100\% suíno; F2 = 25\% caprino/75\% suíno; F3 = 50\% caprino/50\% suíno; F4 = 75\% caprino/25\% suíno; F5 = 100\% caprino. 
Os comentários espontâneos dos provadores nas fichas de aceitação sensorial revelaram que a amostra formulada com $100 \%$ de carne suína, apesar dos comentários positivos em relação ao aroma e sabor, recebeu muitos comentários negativos sobre a aparência devido à falta de cor e palidez do produto. A amostra formulada com $100 \%$ de carne caprina também recebeu comentários negativos em relação à aparência muito escura, sendo o aroma e o sabor considerados desagradáveis. As amostras intermediárias, com diferentes proporções de carnes suína e caprina, receberam comentários positivos e negativos em relação aos atributos aparência, aroma, sabor e textura não evidenciando nenhuma tendência marcante.

\section{CONCLUSÃO}

De acordo com os resultados pode-se concluir que a adição de $25 \%$ de carne caprina na formulação de embutido fermentado, junto com carne suína, foi satisfatória não interferindo na aceitação sensorial do produto. As características microbiológicas dos produtos obtidos atenderam à legislação em vigor e quando estes foram comparados com o produto comercial evidenciaram teores de gordura similares e maiores valores de umidade.

\section{Abstract \\ STUDY OF THE PHYSICAL-CHEMICAL, MICROBIOLOGIC AND SENSORIAL CHARACTERISTICS OF SALAMI TYPE FERMENTED SAUSAGES FORMULATED WITH DIFFERENT PROPORTIONS OF PORK AND GOAT MEAT}

Salami type fermented sausages were formulated with different proportions of pork and goat meat, aiming the use of goat meat in a processed meat product. Influence of $\mathrm{pH}$ and Water Activity (Aw) were evaluated in different formulations. Final products were characterized in relation to moisture, fat and protein contents. Microbiological analysis and sensory acceptance (hedonic scale with 9 points) were also conducted. Goat meat addition in formulations affected final values of $\mathrm{pH}$ and $\mathrm{Aw}$. When compared with a commercial sample, pork/goat meat fermented sausages showed similar values for fat content and moisture values were higher for formulated products. Microbiological counts attended federal laws. Formulation containing $25 \%$ goat meat and $75 \%$ pork meat was considered the best one, which did not show any interference in sensory acceptance of the product.

KEY WORDS: SALAMI, GOAT, PORK, COMPOSITION. 


\section{REFERÊNCIAS}

1 ACTON, J.C.; DICK, R.L. Improved characteristics for dry, fermented turkey sausage. Food Product Development, Chicago, v.9, n.8, p.91-94, Oct., 1975.

2 APHA. American Public Health Association. Compendium of methods for the microbiological examination of foods. $3^{\text {rd }}$ ed. Washington, 1992. $1219 \mathrm{p}$.

3. AOAC. Association of Official Analytical Chemists. Official methods of analysis of AOAC international. $15^{\text {th }}$ ed. Washington, 1990. $1298 \mathrm{p}$.

4 ARGANOSA, F.C.; MANZANO, M.L.; ARGANOSA, V.G. Chemical and organoleptic characteristics of emulsion goat meat sausages containing pork fat or shortening. Philippine Agriculturist, Laguna, v.58, n.9/10, p.356-359, 1975.

5 BACUS, J. Update: meat fermentation. Food Technology, Chicago, v.38, n.6, p.59-69, June, 1984.

6 BERRY, B.W.; CROSS, H.R.; SMITH, G.C. Processing, chemical, sensory and physical properties of bacon-chopped and formed products, made from pork, beef, mutton and chevon. Journal of Muscle Foods, Trumbull, v.1, n.1, p.45-57, Jan., 1990.

7 BÖHME, H.M.; MELLETT, F.D.; DICKS, L.M.T.; BASSON, D.S. Production of salami from ostrich meat with strains of Lactobacillus sake, Lactobacillus curvatus and Micrococcus sp. Meat Science, Essex, v. 44, n.3, p.173-180, Nov., 1996.

8 DEGENHART, J. Tecnologia de produtos curados. Campinas: ITAL, 1988. p. 51-71. (Curso de Tecnologia da Carne).

9 DZUDIE, T.; TANDEM, C. A comparative study of goat, beef and rabbit sausages. Journal of Food Science and Technology, Mysore, v.31, n.4, p.333-334, July/Aug., 1994.

10 GARCIA, A.M. Produção e avaliação de um embutido fermentado contendo carne de aves mecanicamente separada. Viçosa, 1995. 65 p. Dissertação (Mestrado em Ciência e Tecnologia de Alimentos) - Universidade Federal de Viçosa. 
11 HELGESEN, H.; NAES, T. Selection of dry fermented lamb sausages for consumer testing. Food Quality and Preference, Oxford, v.6, n.2, p.109-120, 1995.

12 HWANG, J.W.; ANGLES, S.; KINSMAN, D.M.; HALL, K.N. Preparation of fermented sausages from underutilized fish and meat sources. Journal of Food Processing and Preservation, Trumbull, v.13, n.3, p.187-200, 1989.

13 JUDGE, M.D.; ABERLE, E.D.; FORREST, J.C.; HEDRICK, H.B.; MERDEL, R.A. Principles of meat science. Dubuque: Kendall/ Hunt, 1989. $351 \mathrm{p}$.

14 KLETTNER, P.G.; PÖLLEIN, H.; OTT, G. Processing of old sheep in the meat industry. Fleischwirtschaft, Frankfurt, v.69, n.12, p.18101835, 1989.

15 KRUPA, J.; ZIN, M.; DOMINIK, M. Utilization of goat meat in meat products. Gospodarka-Miesna, Warsaw, v.44, n.4, p.18, 23-25, 1992.

16 MEILGAARD, M.; CIVILLE, G.V.; CARR, B.T. Sensory evaluation techniques. Boca Raton: CRC, 1987. v. 2.

17 MELO, L.R.R. Utilização de carne de caprinos de descarte na fabricação de um embutido cozido, tipo apresuntado. Fortaleza, 1998. 58 p. Dissertação (Mestrado em Tecnologia de Alimentos) Universidade Federal do Ceará.

18 PRICE, J.F.; SCHWEIGERT, B.S. Ciencia de la carne y de los productos carnicos. Zaragoza: Acribia, 1976. $668 \mathrm{p}$.

19 SAS Institute Inc. Statistics analyses systems (SAS): versão 6.12. Cary, NC, 1996.

20 SILVA, R.Z.M. Produtos fermentados acrescidos de proteína não cárnea e carne de frango mecanicamente separada. In: SILVA, R.Z.M. (Ed.). Aplicação da biotecnologia em produtos cárneos. Campinas: ITAL, 1990. p. 151-178.

21 SILVEIRA, E.T.F.; ANDRADE, J. Aspectos tecnológicos de processamento e qualidade de embutidos fermentados. Campinas: FEA/UNICAMP, 1991.

22 TERRA, N.N. ; BRAUN, M.A.R. Carne e seus derivados: técnicas de controle de qualidade. São Paulo: Nobel, 1985. 121 p. 
23 ZAPATA, J.F.F. Tecnologia e comercialização da carne ovina. In: SEMANA DA CAPRINOCULTURA E DA OVINOCULTURA TROPICAL BRASILEIRA, 1., Sobral, 1994. Anais... Brasília: EMBRAPA-SPI, 1994. p. 115-128. 